\title{
Angular Distribution of Secondary Ions under FIB-shave-off Condition -Toward Development of Three-Dimensional Sec- ondary Ion Image System-
}

\author{
Ahsan Habib, ${ }^{1, *}$ Hiroyuki Asakura, ${ }^{1}$ Miku Furushima, ${ }^{1}$ So-Hee Kang, ${ }^{1}$ Yun Kim, ${ }^{1}$ \\ Bunbunoshin Tomiyasu ${ }^{1,2}$ and Masanori Owari ${ }^{1,2}$ \\ ${ }^{1}$ Institute of Industrial Science, The University of Tokyo, 4-6-1 Kobama, Meguro-ku, Tokyo 153-8505, Japan \\ ${ }^{2}$ Environmental Science Center, The University of Tokyo, 7-3-1 Hongo, Bunkyo-ku Tokyo 113-0033, Japan \\ *corresponding.habib@iis.u-tokyo.ac.jp \\ habibchem@du.ac.bd
}

(Received: November 11, 2016; Accepted: May 16, 2017)

\begin{abstract}
In our previous studies, some approaches have been taken to get three-dimensional (3D) image for both inorganic and organic materials using FIB-ToF-SIMS technique. Of these, shave-off cross-sectioning and metal-assisted shave-off processes were predominant. In the present study, a further approach has been taken to get a 3D image through developing 3D shave-off cross-sectioning method for the generation of secondary ions. Currently used shave-off cross-sectioning method is able to provide $2 \mathrm{D}$ image on $\mathrm{X}-\mathrm{Z}$ plane and that can be improved into 3D by using resistive anode detector through changing the optical transportation system of the secondary ions. Installation of cylindrical lens along Z-axis shows 1000 times magnification of the real image on the detector. For investigation of angular distribution of secondary ions, shape and/or angle of the shave-off cross-sectioning areas, using W-wires as model samples, was evaluated. Shave-off method exhibited the formation of a certain shape of the sample surface with $87^{\circ}$ angle. The peak angle of the generated secondary ions was $\sim 45^{\circ}$ for $87^{\circ}$ tilted samples (Si-wafer and Al-foil) and the observed secondary ion images were $2 \mathrm{D}$ by using a resistive anode detector. No significant change in angular distribution of the secondary ions was observed from highly roughness (Al-foil) and almost flat surfaces (Si-wafer).
\end{abstract}

\section{Introduction}

Three-dimensional microanalysis of microstructure for both inorganic and organic materials is important in the development and progress of analytical methods on the micro-to-nanometer scales. Focused ion beam-secondary ion mass spectrometry (FIB-SIMS) has proven as an important analytical tool for determination of two-dimensional spatial distribution of chemical compounds in many materials. Therefore, it is necessary to pay attention how the existing FIB-SIMS can be used, through adopting and/or modification of secondary ion optics, for three-dimensional (3D) microanalysis. Fig. 1 shows a schematic diagram of gallium focused ion beam-secondary ion mass spectrometry (Ga-FIB-SIMS) which provides one-dimensional (1D) secondary ion mass spectra of target materials' compounds and it can be used for measuring of two-dimensional (2D) spatial distribution of secondary ions by introducing a resistive anode detector. 2D secondary ion images can be achieved through coupling the resistive anodic detector with the existing FIB-SIMS (Fig. 1). Prof. Owari's group has taken several approaches to get 3D images not only of inorganic and organic/polymer materials [1-4] but also for biological samples by using the 2D shave-off cross-sectioning FIB-SIMS technique [5]. Fig. 2 shows schematic diagrams of 2D shave-off cross-sectioning method (Fig. 2a) and shave-off depth profiling (Fig. 2b). The shave-off method is advantageous over other 


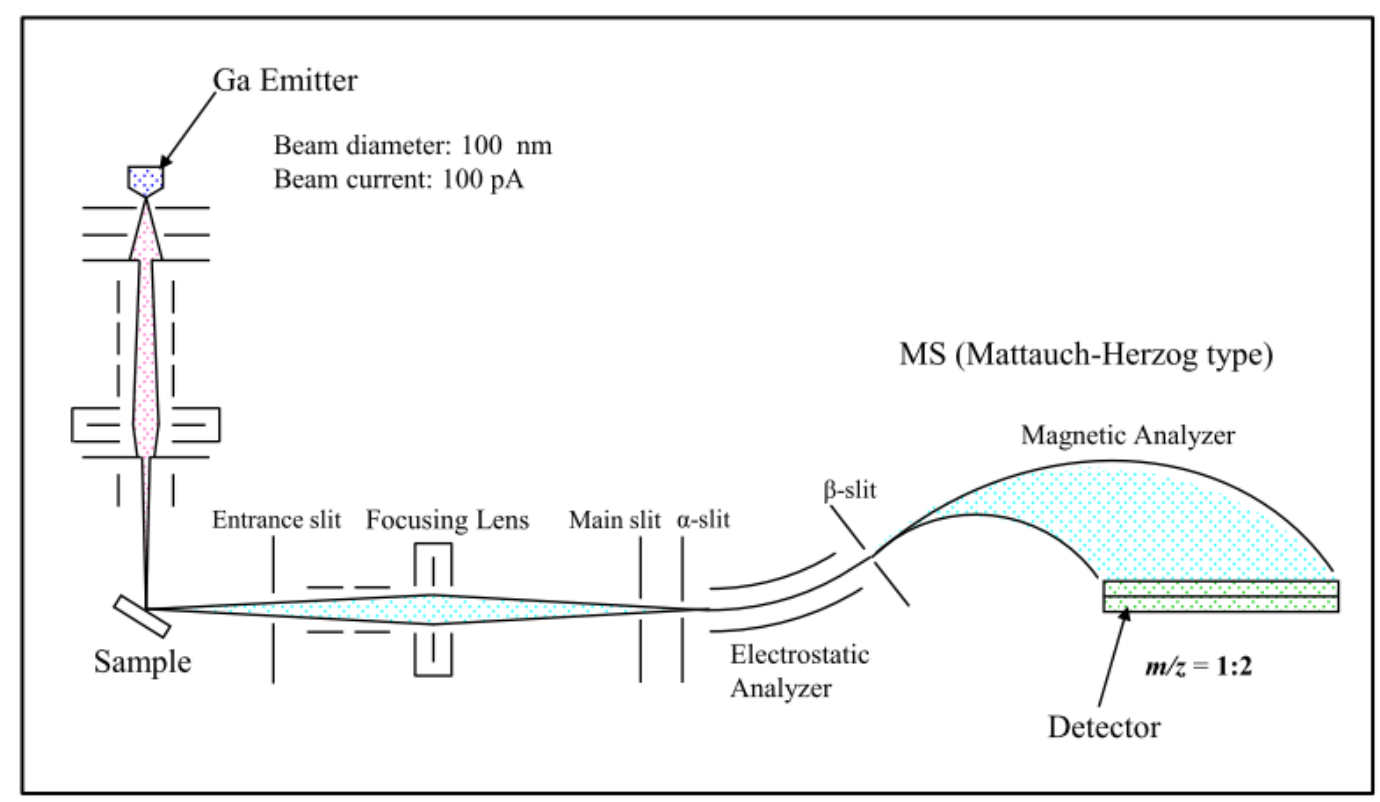

Figure 1. (color online) Schematic diagram of Ga-FIB SIMS apparatus.

micro-machining techniques with respect to surface roughening, and sputtered deposition of the cross-sectioning area. This is due to achieving almost uniform depth profiling under the shave-off scanning mode (A fast horizontal sweep of an FIB is combined with a very slow vertical sweep). Moreover, the FIB of the shave-off method shaves completely off the sample from the surface along the depth direction under dynamic conditions.

Fig. 3 shows an outline of secondary ion transport optical system in 3D shave-off cross-sectioning analysis. Fig. 3a shows the transportation of secondary ions on $\mathrm{X}-\mathrm{Y}$ plane and virtually no magnification effect is observed on the detector (upside). Fig. $3 b$ shows the traveling of secondary ions on the Y-Z plane and observed significant magnification effect by introducing cylindri- cal lens. As shown from Fig. 3c, magnification becomes $1 / 2$ of the real image in front of the cylindrical lens $\mathrm{C} 2$ at position $\mathrm{B}$ (downside), on the other hand, the magnification appears as 1000 times of the real image by using the cylindrical lens $\mathrm{C} 2$ on the detector (upright). This concept will be quite helpful to establish 3D shave-off cross-sectioning method and this new method will provide to develop a 3D secondary ion imaging system for micro-to-nano scale analysis.

In the present study, attempts have been taken to investigate the angular distribution of secondary ions under shave-off cross sectioning condition through investigation of shape/angle of shaved-off cross-sectioning areas. The gained knowledge of the angular distribution of secondary ions has been executed to construct an optical system of transportation of secondary ions that will pro-
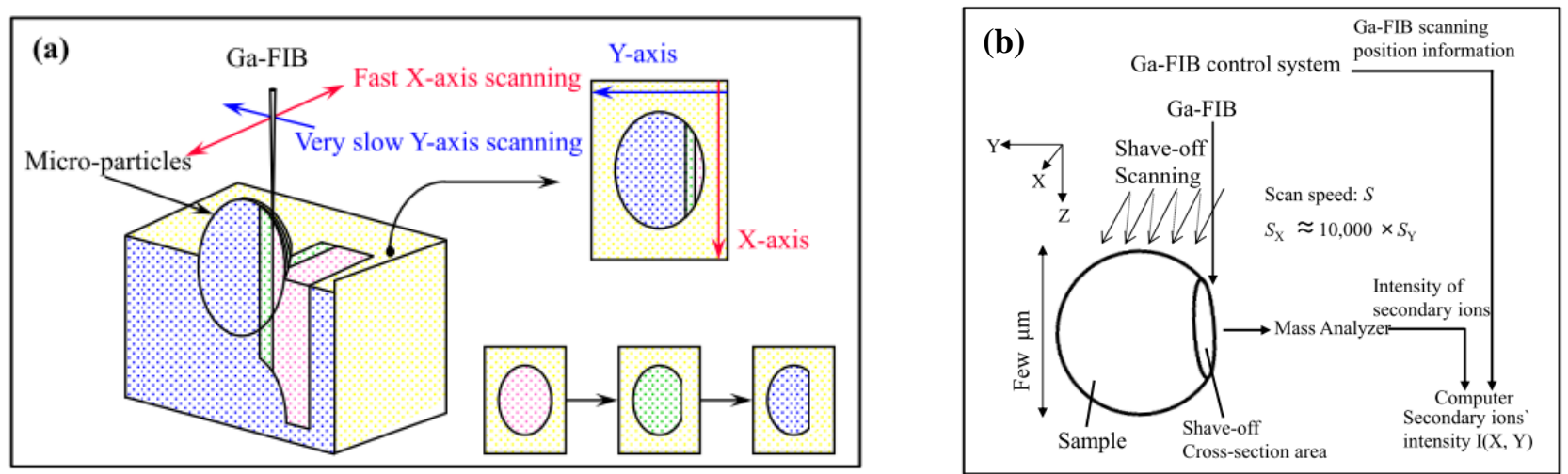

Figure 2. (color online) Schematic diagram of (a) shave-off depth profiling and (b) 2D shave-off cross-sectioning system. 


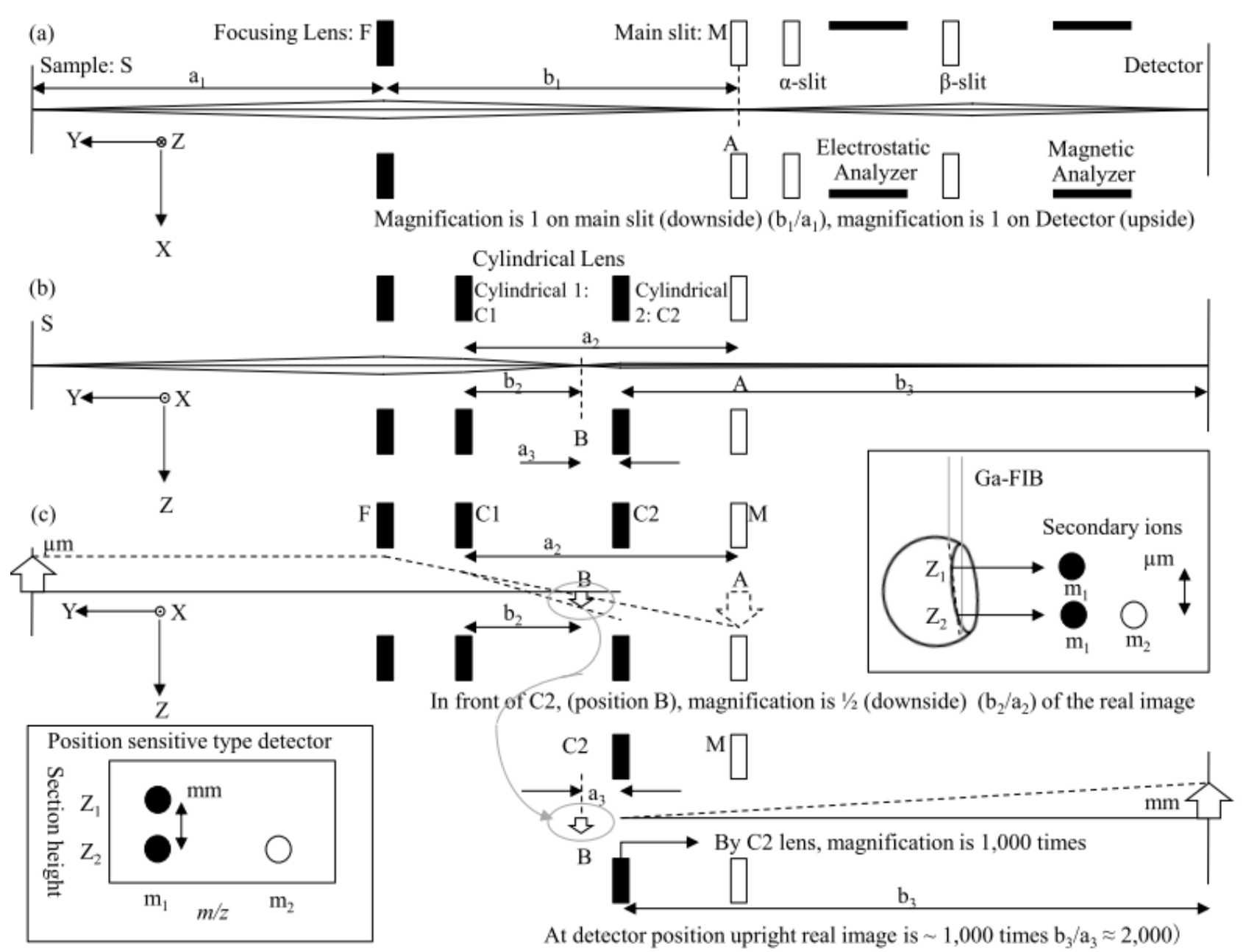

Figure 3. An outline of secondary ion transport optical system in 3D shave-off analysis. 3a, b stand for secondary ions travelling on $\mathrm{X}-\mathrm{Y}$ and $\mathrm{Y}-\mathrm{Z}$ planes, respectively. 3c stands for effect of cylindrical lens on magnification in detector (along Z-axis).

vide the concept of 3D shave-off cross-sectioning analysis. Finally, attention will be paid on the development of 3D secondary ion imaging system.

\section{EXPERIMENTAL}

\subsection{Preparation of shave-off cross-sectioning area} using focused ion beam

About $8 \mathrm{~mm}$ length with $40 \mu \mathrm{m}$ diameter tungsten (W) wire (Nilaco, 99.95\%) was taken as a model material to investigate the shave-off cross sectioning area under nano-Ga-FIB beam (beam current: 250-260 pA; beam diameter: $40 \mathrm{~nm}$ ) by using an SMI3050SE apparatus (manufactured by SII Nanotechnology, now Hitachi High-Tech Science Corporation) at an accelerating voltage of $30 \mathrm{kV}$. TEM images of the shaved-off $\mathrm{W}$-wire were obtained by using a transmission electron microscope (JEM1010, JEOL) as shown in Fig. 4.

\subsection{Measurement of distribution of secondary ion images}

Silicon wafer $(10.65 \times 7.50 \mathrm{~mm})$ of $0.50 \mathrm{~mm}$ thickness and aluminum foil $(8.65 \times 5 \mathrm{~mm})$ of $10 \mu \mathrm{m}$ thickness were taken as model materials to investigate the dependence of the angular distribution of secondary ions from $87^{\circ}$ tilted samples under raster scanning mode. The detector was adjusted about $6 \mathrm{~mm}$ far from the sample stage and downed about $14 \mathrm{~mm}$ of its center from the sample stage. Such an arrangement is shown in Fig. 5a. The Ga-FIB was generated at accelerated voltage $20 \mathrm{kV}$, and its beam diameter and beam current were $\sim 1 \mu \mathrm{m}$ and $\sim 100 \mathrm{pA}$, respectively. The Ga-FIB was used for obtaining the secondary ion images by using a resistive anode type detector coupled with an oscilloscope and a position computer. 

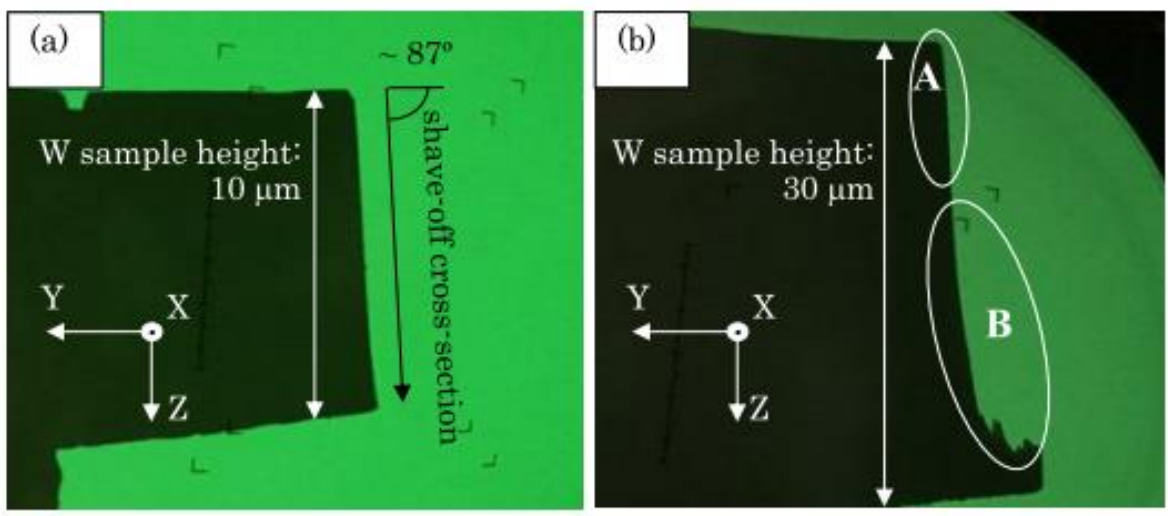

Figure 4. (color online) TEM observation of shaved-off cross-sectioning areas made by using FIB micromachining process of different heights (a) $10 \mu \mathrm{m}$ and (b) $20 \mu \mathrm{m} \mathrm{W}$-wires.

\section{RESULTS AND DISCUSSION}

It has been reported that shave-off cross-sectioning is an efficient micro-machining method to achieve highly precise depth profiling with nanometer-dimensional depth resolution, which would provide spatial distribution of materials compounds [3,4]. Different heights of two $\mathrm{W}$-wires were taken as model material to investigate their shave-off cross-sectioning shape and/or angle by using Ga-FIB micro-machining method. This is because the angular distribution of secondary ions depends on the shape and/or angle of the shave-off cross-sectioning area. Fig. 4 shows the TEM images of two different heights W-wires after micromachining by Ga-FIB. Fig. 4a shows the TEM image for $10 \mu \mathrm{m}$ height $\mathrm{W}$-wire where a certain shape of the cross-sectioning area and its angle are ob- served. The angle of the shape is about $87^{\circ}$. On the other hand, Fig. 4b shows the TEM image for $30 \mu \mathrm{m}$ height W-wire where two distinct parts of the Ga-FIB cross-sectioning areas are observed. The parts are designated as part-'A' and part-' $B$ '. Part-' $A$ ' indicates the shape of FIB cross-sectioning area and that just superimpose of the shape of shave-off cross-sectioning area for $10 \mu \mathrm{m} \mathrm{W}$-wire (Fig. 4a). The shape for part-'B' also shows the same angle $\left(87^{\circ}\right)$ that is observed for $10 \mu \mathrm{m}$ W-wire (Fig. 4a). However, part 'B' (Fig. 4b) showing completely different shape from part ' $\mathrm{A}$ ', and the following observation may be taken into account: (i) about the scattered $\mathrm{Ga}^{+}$primary ions that leads scattered angle and (ii) about the re-deposition of emitted W neutral particles with high kinetic energy. Such observations might
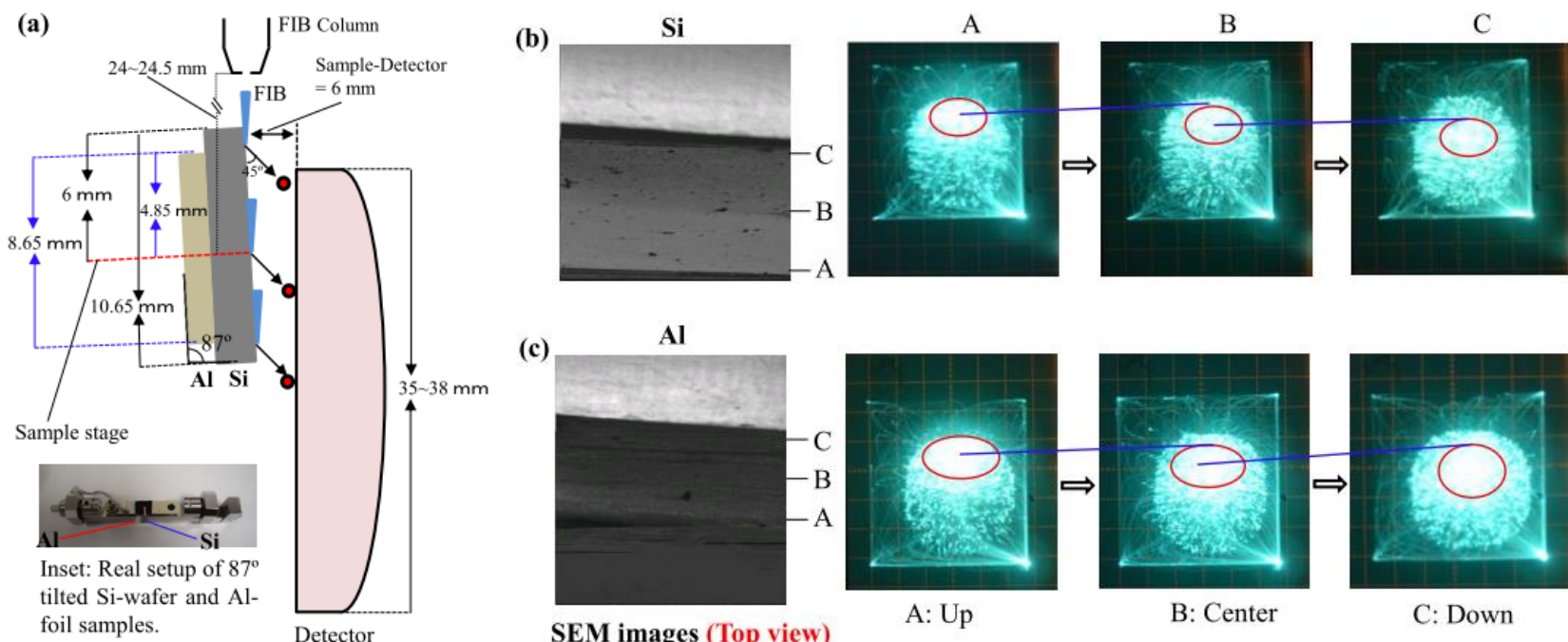

(c)
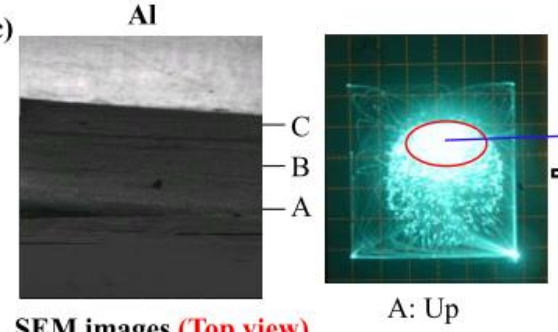

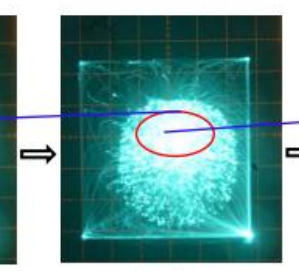

B: Center

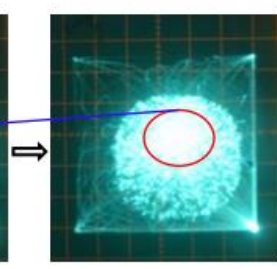

C: Down

Figure 5. (color online) The samples were adjusted at $87^{\circ}$ with the normal angle of FIB for recording the secondary ion images (a). Ion induced secondary electron images of silicon wafer (b) and aluminum foil (c). Inset: Real setup of $87^{\circ}$ tilted Si-wafer and Al-foil samples. 
be helpful for further development of Ga-FIB-based micromachining method. It is, therefore, concluded that the Ga-FIB acts as shave-off cross-sectioning micromachining method within a certain depth (e.g., $10 \mu \mathrm{m})$ of target materials.

To investigate the dependency of the angular distribution of the secondary ions on the shaved-off shape/angle, the target materials, Si-wafer and Al-foil, were tilted at $87^{\circ}$ with respect to the sample stage as shown in Fig. 5a. Inset in Fig. 5a shows the real $87^{\circ}$ tilted $\mathrm{Si}$ and $\mathrm{Al}$ samples. This geometry allows scanning of the sample surface by the incident Ga-FIB at $3^{\circ}$ which corresponds to the angle of shaved-off W-wire. Figs. $5 \mathrm{~b}$ and $5 \mathrm{c}$ show the total secondary ion images of Si-wafer and Al-foil samples, respectively. $\mathrm{A}, \mathrm{B}$ and $\mathrm{C}$ indicate the scanning positions of the samples, and A for several $\mu \mathrm{m}$ below from the top edge, $\mathrm{B}$ corresponds to the center and $\mathrm{C}$ for several $\mu \mathrm{m}$ above from the bottom edge. Results show that the secondary ions emitted from the sample surfaces with about $45^{\circ}$ with respect to the horizontal axis of the sample stage. At position A (just below the top edge), the highly intense spot of the secondary ions appears at the top of the detector and that gradually goes to downwards to the detector for positions B (center) and $\mathrm{C}$ (just above the bottom edge). As shown from Figs. $5 b$ and $5 c$, the intensity of the secondary ions spot of Al-foil is significantly intense compared to those for $\mathrm{Si}$-wafer. This is because of higher ionization efficiency of aluminum and that also provides high ion yield. Although the roughness of Al-foil is at $\mu \mathrm{m}$ level whereas that for Si-wafer is at $\mathrm{nm}$ level, no significant change in angular distribution was observed from these two samples under the present experimental conditions. Variation of the angular distribution of secondary ions with the changing of tilt angles of different samples has also been attempted. Further attempt for investigation of development of 3D secondary ion imaging system by using 3D shave-off cross-sectioning method has already been taken and that in progress in our laboratory.

\section{CONCLUSION}

In this work, the shape and/or angle of shaved-off cross-sectioning areas of $\mathrm{W}$-wires were evaluated by TEM images for investigation of angular distributions of secondary ions by using Ga-FIB micromachining method. A certain shape with $87^{\circ}$ angle was observed from $10 \mu \mathrm{m}$ height $\mathrm{W}$-wire while $30 \mu \mathrm{m}$ height $\mathrm{W}$-wire shows two distinct Ga-FIB cross-sectioned parts. The upper part with about $10-12 \mu \mathrm{m}$ depth exhibits a shape with $87^{\circ}$ angle which are identical with those for $10 \mu \mathrm{m}$ height W-wire. Ga-FIB cross-sectioning acts as a shave-off cross-sectioning method with shallow depth $(\sim 10-12 \mu \mathrm{m})$ of the target materials. The angle of the generated secondary ions was $\sim 45^{\circ}$ for $87^{\circ}$ tilted samples ( $\mathrm{Si}$-wafer and Al-foil) and the observed secondary ion images were 2D by using a resistive anode detector. No significant change in the angular distribution of the secondary ions was observed from highly roughness (Al-foil) and almost flat surfaces (Si-wafer). An outline for optical transport of the secondary ions has been proposed on the basis of the angular distribution of secondary ions that will provide $3 \mathrm{D}$ shave-off cross-sectioning concept by introducing cylindrical lens along Z-axis. By using this new 3D shave-off cross-sectioning method coupled with FIB-SIMS, 3D imaging system will be achieved.

\section{Acknowledgement}

This research was supported in part by a research fellowship of the Japan Society for the Promotion of Science (JSPS) for visiting Scientists (L15529).

\section{References}

[ 1] H. Satoh, M. Owari and Y. Nihei, J. Vac. Sci. Technol. B 6, 915 (1988).

[ 2] Y. Kim, Y. Morita and M. Owari, J. Surf. Anal., 17, 337 (2010).

[3] A.Yamazaki, S. Akiba, B. Tomiyasu and M. Owari, e-J. Surf. Sci. Nanotech., 13, 65 (2015).

[ 4] M. Toi, A. Maekawa, T. Yamamoto, B. Tomiyasu, T. Sakamoto, M. Owari, M. Nojima, and Y. Nihei, J. Surf. Anal. 12, 170 (2005).

[ 5] T. Iwanami, Y.J. Liu, M. Okazaki, M. Nojima, T. Sakamoto and M. Owari, Surf. Interface Anal. 38, 1658 (2006).

[ 6] H. Asakura, A. Habib, M. Furushima, S-H. Kang, Y. Kim, B. Tomiyasu and M. Owari, J. Surf. Anal. (submitted). 